\title{
Pure tone overstimulation changes the micromechanical properties of the inner hair cell stereocilia
}

\author{
Barbara Canlon ${ }^{1}$, Josef Miller ${ }^{3}$, Åke Flock ${ }^{1}$ and Erik Borg ${ }^{1.2}$ \\ ${ }^{\prime}$ Karolinska Institute, Physiology Department II, Stockholm. Sweden, 'Karolinska Sjukhuset, Audiology Department. \\ Stockholm. Sweden and 'Kresge Hearing Research Institute, University of Michigan. Ann Arbor, U.S.A.
}

(Received 24 October 1986; accepted 13 May 1987)

\begin{abstract}
The effect of permanent noise-induced hearing loss on the auditory brainstem response (ABR) and the micromechanical properties of cochlear hair cell stereocilia in guinea pigs was investigated. The threshold of movement of the stereocilia was measured by applying force from a fluid filled pipette. After exposure to a $1.0 \mathrm{kHz}$ pure tone signal at $105 \mathrm{~dB}(\mathrm{~A}) \mathrm{for} 72 \mathrm{~h}$ the threshold of the ABR was broadly elevated by approximately $50 \mathrm{~dB}$. Inner hair cell stereocilia showed a decrease in threshold while the outer hair cell stereocilia bundles remained unaltered. This effect was localized to the $13-15 \mathrm{~mm}$ distance from the stapes corresponding to the region of maximal stimulation. The effect was recorded within $1 \mathrm{~h}$ of exposure and remained constant with exposures up to 7 days. Following a one month recovery period from sound exposure, normal threshold values of stereocilia movement were observed, indicating recovery. At this time. swelling of the afferent dendrites beneath the inner hair cells was observed throughout the cochlea together with approximately $30 \%$ scattered loss of outer hair cells in the 13 to $15 \mathrm{~mm}$ region. The ABR showed some recovery (approximately $20 \mathrm{~dB}$ ), yet a threshold shift remained.
\end{abstract}

Stcroocilia mechanics; Acoustic trauma; ABR threshold; Permanent threshold shift; Temporary threshold shift

\section{Introduction}

The effects of acoustic overstimulation on hearing involves a wide array of insults as revealed in both cochlear physiology and morphology. Behavioral studies in man and animals have characterized changes in threshold sensitivity, discrimination, and selectivity functions with noise trauma, (Davis et al., 1946; Borg and Engström, 1983) while electrophysiological studies have demonstrated eighth nerve changes in threshold and tuning sensitivity and shape (Liberman and Kiang, 1978; Liberman and Dodds, 1984). However, many features of the specific relationships between pathophysiology and histopathology after noise-induced hearing loss must yet he determined.

One area of basic and clinical importance concerns the relationship between temporary (TTS)

Correspondence to: B. Canlon, Karolinska Institute, Physiology Department II, S-104 01 Stockholm, Sweden. and permanent (PTS) hearing loss and the extent to which the stereocilia influence these changes in auditory sensitivity. It has been demonstrated that selective stereocilia lesions resulting from a permanent noise-induced hearing loss leads to specific auditory sensitivity changes (Robertson, 1982; Slepecky et al., 1982; Engström et al., 1983; Libermann and Dodds, 1984). Recent studies suggest that the tip-links between the stereocilia are sensitive to acoustic trauma and that these links are required for transduction (Pickles et al., 1987). However, a permanent threshold shift is accompanied by a certain degree of cell death and degeneration making interpretations of the specific role of stereocilia difficult. In addition, fixation artifacts preclude conclusive interpretations regarding the 'stiffness' characteristics of pathological stereocilia. Micromechanical measurements of stereocilia after acoustic overstimulation are needed in order to establish a more direct relationship between stereocilia pathophysiology and changes in auditory function. 
In previous publications, direct observations and measurements of the micromechanical behavior of the stereocilia have been made on isolated coils from the guinea pig cochlea (Strelioff and Flock, 1984; Saunders and Flock, 1986; Saunders et al., 1986a, b). The threshold of stereocilia motion has been shown to vary with hair cell row and in an apico-basal direction along the cochlea. Among the outer hair cells, the stereocilia of the first row cells are the most stiff followed by the second, then the third row. Inner hair cell stereocilia exhibited a stiffness value similar to the second row outer hair cells.

The purpose of the present study was to measure the micromechanical properties of the cochlear hair cell stereocilia after exposing guinea pigs to a stimulus known to yield a PTS and to determine the associated shift of the frequencyspecific auditory brainstem response thresholds.

\section{Materials and Methods}

Sixty-five Hartley strain pigmented guinea pigs $(200-350 \mathrm{~g})$ were used in this study. The general protocol of the experiment was: (1) measure the frequency-specific auditory brainstem response (ABR) threshold sensitivity; (2) expose to sound; (3) repeat ABR measurements; and (4) perform micromechanical measurements on stereocilia bundles.

The majority of animals were exposed bilaterally to a $1.0 \mathrm{kHz}$ pure tone for $72 \mathrm{~h}$ and $A B R$ measurements were made immediately following the exposure. ABR measurements required approximately $1 \mathrm{~h}$. The preparation of the cochlea for stereocilia measurements required $20-40 \mathrm{~min}$. Thus, micromechanical measures were initiated approximately $1.5 \mathrm{~h}$ after the exposure ended.

\section{ABR measurements}

ABR thresholds were determined under Dridol (10 $\mathrm{mg} / \mathrm{kg}$ body $\mathrm{wt}$ ) and pentobarbitol anesthesia ( $25 \mathrm{mg} / \mathrm{kg}$ body wt) delivered intraperitoneally. Responses were differentially recorded between subcutaneous stainless steel electrodes at the vertex, and the ipsilateral retro-auricular region, while the lower back served as ground. The stimuli were single full cycle sine waves filtered through a one-third octave band pass filter with the corre- sponding center frequency from 0.4 to $16 \mathrm{kHz}$. Stimuli were presented through a $12 \mathrm{~cm}$ tube connected to an earphone (Telephonics TDH 39) and sealed to the external auditory canal. Electrical activity was amplified 10000 times and averaged by a signal averager (Medlec MS 92). Stimulus intensity was calibrated with a $6.25 \mathrm{~mm}$ condenser microphone (Brüel and Kjaer type 2607) using pure tones. All sound pressure levels (SPL) were expressed in $\mathrm{dB}$ relative to $20 \mu \mathrm{Pa}$. The intensity of the stimulus was varied in $5 \mathrm{~dB}$ steps and adjusted until a visual detection threshold (VDT) was obtained. The VDT was defined on the basis of a number of repetitive measurements made for stimuli at each frequency. Testing was performed in a double-wall sound attenuated booth.

\section{Sound exposure}

Unanesthetized animals were sound exposed in an open field acoustic chamber $(225 \times 120 \times 100$ $\mathrm{cm})$. The ceiling and walls were made of perforated masonite plates backed with acoustic absorption material. The loud speaker was placed 70 cm above the wire mesh animal cage $(55 \times 32 \times 20$ $\mathrm{cm})$. Sound calibration was performed within the wire mesh cage with a $12.5 \mathrm{~mm}$ condenser microphone (Briel and Kjaer model 2213). The exposure intensity used in this investigation was 105 $\mathrm{dB}(\mathrm{A})$. The resulting sound field in the exposure chamber varied from 100 to $110 \mathrm{~dB}(\mathrm{~A})$. A $1.0 \mathrm{kHz}$ exposure was used in order to create a third turn lesion.

Exposure and measurement conditions were: (1) bilateral exposure for $1,24,72,120$, or $168 \mathrm{~h}$ followed by stereocilia measurements; $(2)$ bilateral exposure for $72 \mathrm{~h}$, a six-week recovery period, and stereocilia measurements; (3) monaural exposure for $72 \mathrm{~h}$ and stereocilia measurements made immediately after exposure.

Animals were exposed one or two at a time and ad lib access to food and water was provided. Whether animals were exposed singularly or in pairs did not effect either the ABR or the micromechanical results.

\section{Micromechanical measurements}

Micromechanical measurements were made at selected sites from turns 2,3 , or 4 of different 
cochleas. Previous publications have described the procedure in detail (Flock and Strelioff, 1984; Saunders et al., 1986a). After ABR measurements the bullae were rapidly removed, opened and placed in culture media L-15 from Gibco (Leibovitz, 1963). The apex and the hook region were opened and culture medium was gently infused into the perilymphatic space. The lateral wall from the apex to the desired turn was removed and after the appropriate cuts were made through the basilar membrane and modiolus, the coil was removed and mounted in a miniature vise. The preparation was then transferred to a fluid chamber mounted on the stage of the Zeiss interference contrast microscope equipped with a $40 \times$ water immersion objective and viewed at a magnification of $800 \times$. A micromanipulator was used to position a culture medium filled micropipette (7-9 $\mu \mathrm{m})$ perpendicular to and approximately $5 \mu \mathrm{m}$ from the stereocilia bundle to be studied. The pipette was connected to a hydraulic drive system set at a resonance frequency of $200 \mathrm{~Hz}$ (Brüel and Kjaer type 4810 ). The strobe frequency of the stroboscopic light system (Chadwick and Helmuth model 236) was offset a few Hertz from the hydraulic system so that the motion of the stereocilia bundle could be detected. The amplitude of the fluid oscillation from the pipette was controlled by an attenuator that was graded in $1.5 \mathrm{~dB}$ steps. Each visual detection level (VDL) of threshold on stereocilia movement was taken 5 times from each hair cell and 3-5 different cells were studied at each specific location. In order to control for experimental bias, measurements were frequently recorded by one observer while the applied force was varied by a second observed with no knowledge of the readout.

Due to variations in pipette diameter, the absolute driving force varied between experiments and it was therefore necessary to normalize the data. When indicated, the different stereocilia types were normalized to the $\mathrm{OHC}-1$ threshold value. The stereocilia were normalized to the OHC-1 stereocilia from the same location and the same experimental situation. All measurements were made at room temperature.

\section{Results}

\section{$A B R$ measurements}

An example of the thresholds observed to filtered clicks with center frequencies from 0.4 to $16 \mathrm{kHz}$ are shown (Fig. 1). The ABRs to these signals demonstrated the conventional positive peaks 1 to $4-5$ in the first $10 \mathrm{~ms}$ following the acoustic stimulus. The mean value and standard error of the mean of the threshold responses $(\mathrm{dB}$ SPL) for frequencies between 0.4 and $16 \mathrm{kHz}$ are shown (Fig. 1). The values are based upon observations on 11-48 animals at each of the frequencies examined. Thresholds were most sensitive in the $2-8 \mathrm{kHz}$ frequency range. Based upon measurements made during a 1-h period following the exposure $(72 \mathrm{~h}, 1 \mathrm{kHz}, 105 \mathrm{~dB}(\mathrm{~A}))$, the threshold was elevated in the frequency region between 1.0 and $8.0 \mathrm{kHz}$ by $40-50 \mathrm{~dB}$. Each data point is based upon measurements in 4-28 animals. When the exposure duration was progressively increased (up to 7 days) the ABR thresholds showed a systematic loss in hearing sensitivity.

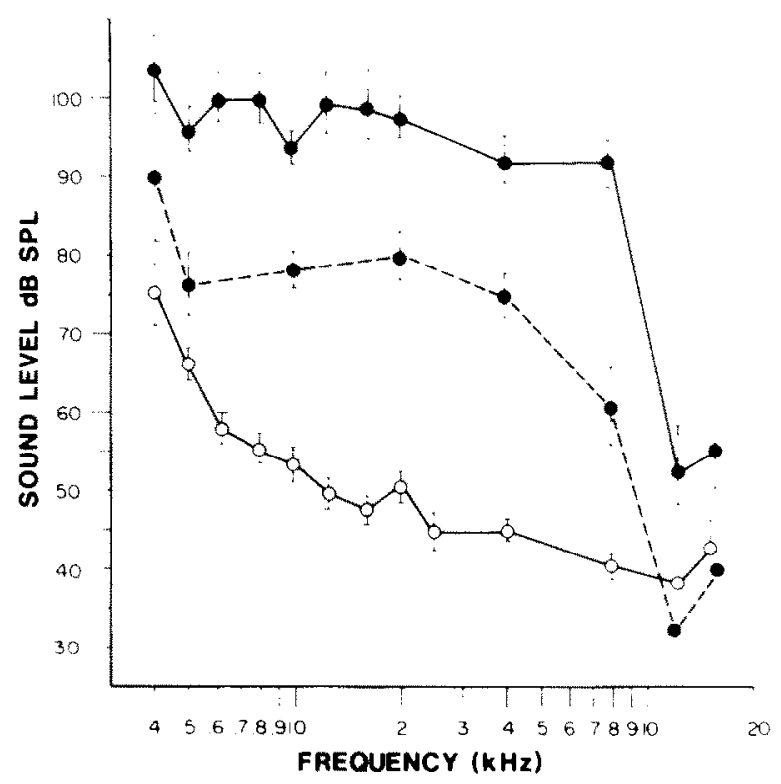

Fig. 1. Threshold curves of the ABR for the guinea pig. All values are the means $\pm S . E$. for pre-exposure thresholds $(0-0, n=11-48) ; \quad$ immediately post-exposure thresholds (- $n=4-28$ ); and 5 weeks post-exposure thresholds $(-\ldots, n=3-11)$ to $1 \mathrm{kHz}, 105 \mathrm{~dB}(\mathrm{~A}), 72 \mathrm{~h}$ of duration. 
The dashed line in Fig. 1 illustrates the findings in 3 -11 subjects permitted a five-week recovery interval following the exposure. A partial recovery of the ABR threshold shift resulting from exposure was found for all frequencies, yet a PTS was evident.

\section{Micromechanical measurements}

When all the data from all regions of the cochlea that were studied (13-17-mm distance from the stapes) were compiled, a total of 232 control cells and 311 noise-exposed cells were measured from 65 guinea pigs. Fig. 2 shows the VDL threshold value for stereocilia bundles from the inner hair cells (IHCs) and the second and third row outer hair cells (OHCs) in control animals and animals tested $1.5 \mathrm{~h}$ after the exposure. All control measurements are normalized to the control OHC-1 cells and the post-exposure measurements are normalized to their respective post-exposure OHC-1 cells. The measurements are from the $15-\mathrm{mm}$ distance from the stapes. The threshold for the control IHC stereocilia was approximately $10 \mathrm{~dB}$ less than the value of the first row $\mathrm{OHC}$ stereocilia.

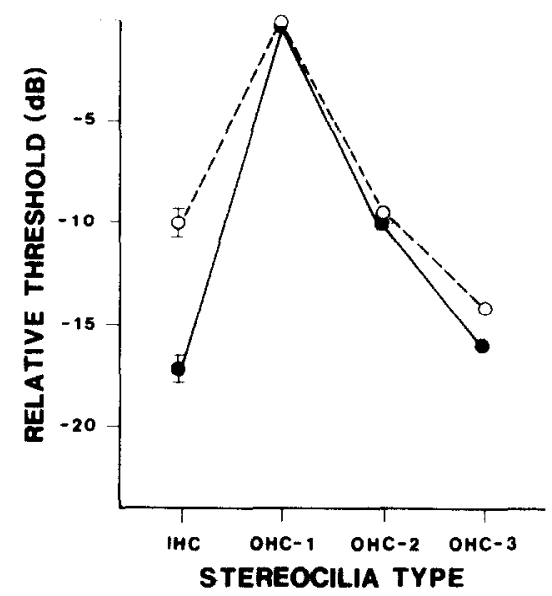

Fig. 2. The effect of a $1.0 \mathrm{kHz}$ pure tone at $105 \mathrm{~dB}(\mathrm{~A})$ for $72 \mathrm{~h}$ on stereocilia micromechanics. The minimal force required to detect stereocilia movement is represented in $\mathrm{dB}$ (re threshold for OHC-1) for the control group (O. . . .,$n=11$ animals) and the group exposed to the pure tone (- $n=7$ animals). Each group has been normalized to their respective mean value of $\mathrm{OHC}-1$ stereocilia within the $15-\mathrm{mm}$ region from the stapes. The S.E. for only IHCs is shown. The S.E. for control $\mathrm{OHC}-2$ is 2.2 and noise $\mathrm{OHC}-2$ is 1.5 . The S.E. for control $\mathrm{OHC}-3$ is 2.3 and noise $\mathrm{OHC}-3$ is 1.8 .
The threshold of the second row $\mathrm{OHC}$ stereocilia was approximately equal to that observed for IHCs, and the threshold of the third row $\mathrm{OHC}$ stereocilia was approximately $14 \mathrm{~dB}$ less than that of the first row $\mathrm{OHC}$ of the control animals. The difference in stereocilia threshold among these control hair cells of different rows in this region of the cochlea corresponds well with previous studies from this laboratory (Saunders et al., 1986a) as well as from studies using calibrated quartz fibers to deflect the stereocilia (Strelioff and Flock, 1984).

In exposed cochleae, a statistically significant $(P \leqslant 0.001)$ decrease in the threshold of the IHC stereocilia (approximately $7 \mathrm{~dB}$ ) was observed compared to the controls. In other words, the IHC stereocilia become less stiff after noise exposure. The threshold for the three rows of OHC stereocilia was similar for both groups. The finding that only the IHC stereocilia change their threshold after stimulation is supported by three additional sets of data. The first is when attenuation values are compared from the same region of both cochleae, using the same pipette, in subjects exposed monaurally. The $\mathrm{OHC}$ stereocilia threshold values ( $\mathrm{dB}$ of attenuation) in the plugged ear were similar to those of the exposed ear, whereas the IHC stereocilia of the exposed ear showed a threshold shift of approximately $14 \mathrm{~dB}$ (Fig. 3). Furthermore, when the $13-15-\mathrm{mm}$ region values of the unexposed and the exposed ears were compared with pipettes of equal diameter, the threshold of the $\mathrm{OHC}$ stereocilia were similar, while the threshold of the noise exposed IHC stereocilia decreased. Finally, when the values in exposed cochleae from the 13-15-mm region were compared to those made at a $17-\mathrm{mm}$ site in the same subject, with the same pipette, the threshold of the OHC stereocilia was comparable to those observed in unexposed tissue and appropriate for the particular basilar membrane site, while the IHC stereocilia threshold showed a change in the $15-\mathrm{mm}$ region and no change in the $17-\mathrm{mm}$ region.

Fig. 4 illustrates the normalized IHC stereocilia threshold values at different sites along the basilar membrane after the exposure. A change in the threshold is seen in the 13-, 14- and 15-mm region whereas the IHC stereocilia in the more apical regions (16- and $17-\mathrm{mm}$ ) are not affected.

Fig. 5 shows the effect of variations in duration 


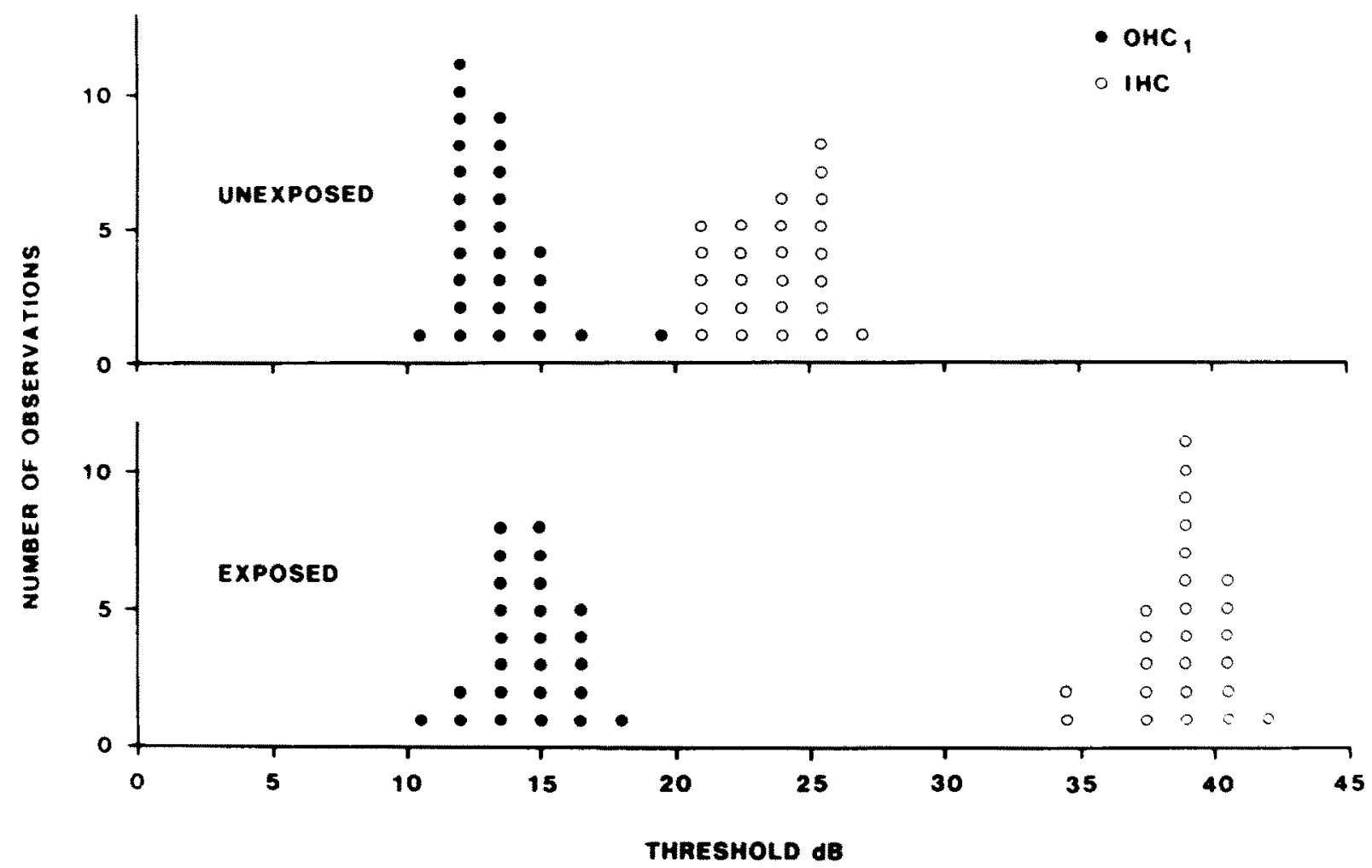

Fig. 3. The effect of monaural exposure $(1 \mathrm{kHz}, 105 \mathrm{~dB}(\mathrm{~A}), 72 \mathrm{~h})$ on the threshold (dB of attenuation) for $1 \mathrm{HC}$ and $\mathrm{OHC}-1 \mathrm{stereocilia}$ movement. The number of observations for each hair cell stereocilia type is shown for the unexposed and exposed ears. The $\mathrm{OHC}$ stereocilia threshold value in the unexposed and exposed ears are similar while the IHC stereocilia threshold value decreased (less stiff) after exposure by $14 \mathrm{~dB}$.

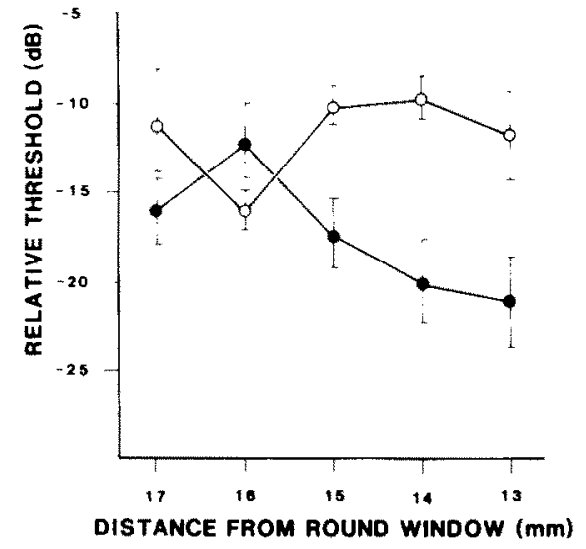

Fig. 4. The localization of IHC stereocilia changes along the length of the basilar membrane after exposure $(1 \mathrm{kHz}, 105$ $\mathrm{dB}(\mathrm{A}), 72 \mathrm{~h})$. The mean and S.E. values for IHC stereocilia threshold are shown (re OHC-1 stereocilia). The control stereocilia are represented by opened circles and data for the 13-, 14-, 15-, 16- and 17-mm distance from the stapes is based on 4, $1,11,2$ and 4 guinea pigs, respectively. The experimental group of the $1 \mathrm{kHz}$ tone exposure on the IHC stereocilia threshold measurements in the 13-, 14- and 15-mm region. In this region, the mean threshold and standard deviation of the normalized control IHC stereocilia is shown by the horizontal solid bar having a value of approximately $10 \mathrm{~dB}$. After exposure durations varying from $1 \mathrm{~h}$ to $7 \mathrm{~d}$. the mean threshold was found to have decreased to $20-25 \mathrm{~dB}$. These data indicate that the effect of sound exposure on the threshold of IHC stereocilia has a relatively rapid onset and appears to remain relatively stable with longer exposures.

In the animals permitted to survive for $840 \mathrm{~h}$ (5 weeks) following exposure to the $1 \mathrm{kHz}$ tone for

is represented by filled circles and data for the 13, 14-, 15-, 16and $17-\mathrm{mm}$ region is based on $5,6,7,5$ and 4 guinea pigs. respectively. Stereocilia measurements from 4 to 6 cells from each location have been made. 


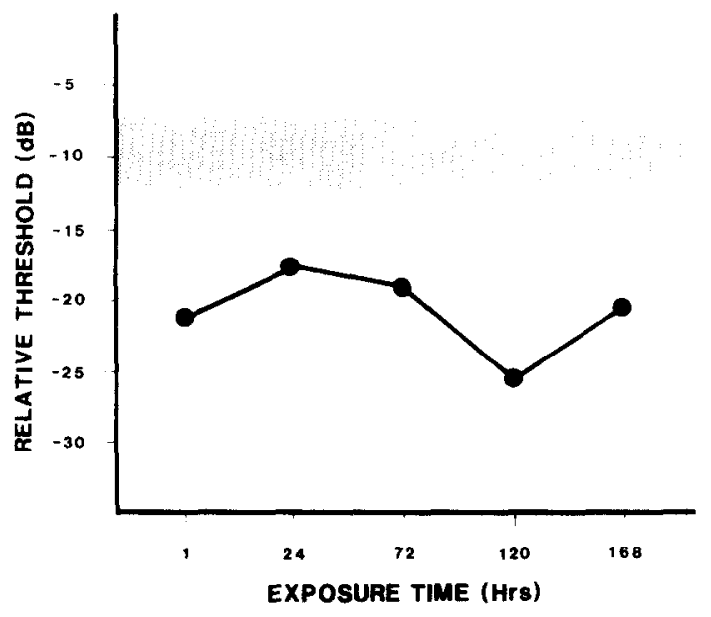

Fig. 5. The effect of exposure duration on IHC stereocilia micromechanics. The mean control value together with the standard deviation is represented by the solid line ( $n=62$ cells from 16 animals). The mean value for the experimental group is shown as a solid circle. The exposure durations were: $1 \mathrm{~h}$ ( $n=20$ cells from 4 animals); $24 \mathrm{~h} \quad(n=15$ cells from 3 animals); $72 \mathrm{~h}$ ( $n=76$ cells from 18 animals); $120 \mathrm{~h}(n=10$ cells from 2 animals); and $168 \mathrm{~h}$ ( $n=5$ cells from 1 animal). The data are compiled from the 13-, 14- and 15-mm region from the round window.

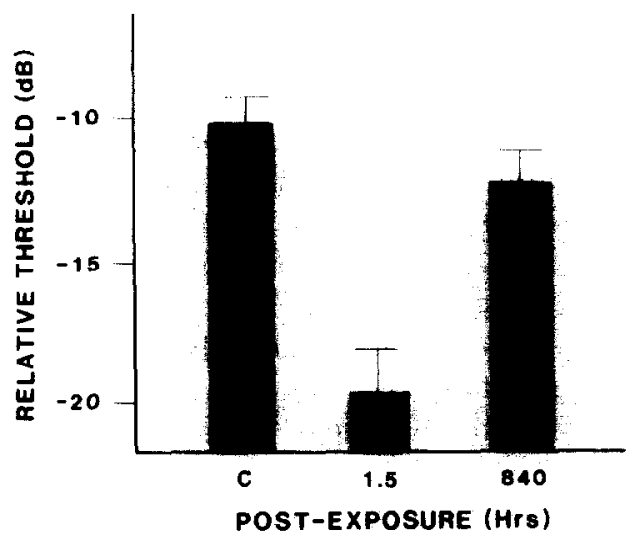

Fig. 6. The recovery with time of the IHC stereocilia micromechanics. The mean control value (C) and the standard deviation are shown ( $n=62$ cells from 16 animals); with values obtained $1.5 \mathrm{~h}$ post-exposure $(1 \mathrm{kHz}, 105 \mathrm{~dB}(\mathrm{~A}), 72 \mathrm{~h})$ ( $n=76$ cells from 18 animals); and after 840 h ( 5 weeks) post-exposure ( $n-48$ cells from 3 animals). Measurements were made in the 13-, 14-, and 15-mm distance from the stapes. After a five-week recovery period the threshold for IHC stereocilia is shown to be within the range of the control threshold. Each bar represents the mean value with the S.E.
$72 \mathrm{~h}$, the IHC stereocilia were found to recover (Fig. 6). The control value $(C)$ of the normalized IHC stereocilia (re $\mathrm{OHC}-1$ ) are shown in the first solid bar with a threshold of $-10 \mathrm{~dB}$. After exposure the threshold is decreased by $9 \mathrm{~dB}$, and with a $840 \mathrm{~h}$ recovery period, the IHC stereocilia threshold approaches control values. At this time scattered $\mathrm{OHC}$ loss with mature scars were observed in the $13-15-\mathrm{mm}$ region of the isolated coils. All but an occasional IHC were intact and all remaining hair cells had normal appearing stereocilia. Light microscopic observations of cross-sections through the organ of Corti have shown swelling of the afferent dendrites beneath the IHC throughout the second, third and fourth turns of the cochlea.

\section{Discussion}

For the region most frequently measured in the present study (15-mm), the control IHC and the three rows of $\mathrm{OHC}$ stereocilia exhibited a similar threshold pattern to those of the previously mentioned studies (Strelioff and Flock, 1984; Saunders et al., 1986a, b). Following acoustic stimulation, the threshold of the IHC stereocilia decreased. These IHC stereocilia bundles required less force in order to initiate movement. Interestingly, no structural alteration of the stereocilia bundles was obvious. The finding that IHC stereocilia are more sensitive to noise trauma than the $\mathrm{OHC}$ stereocilia has been previously reported (Engström and Borg, 1981; Thorne et al., 1984; Engström et al., 1986). These authors have also demonstrated that hair cell body damage induced by noise is primarily an OHC phenomena, which is consistent with our observations. The greater susceptibility to damage of the IHC stereocilia may thus be reflected in any of the microstructural features that differ between IHC and OHC stereocilia bundles. These structural sites include the stereocilium core, their tiplinks, or the cuticular plate region. It is not known which of these regions is most susceptible to noise, and it remains to be determined at the electron microscopic level which sites are responsible for the change in threshold.

Another possible mechanism may involve interactions between the rootlet of the stereocilia and the cuticular plate. It has been shown that the 
organization of actin filaments around the perimeter of the cuticular plate forms a ring-like structure (Hirokawa and Tilney, 1982). In this region there are also actin filaments of opposite polarity (Slepecky and Chamberlain, 1982). The extent to which this ring-like structure plays a role in controlling the threshold of the cochlear stereocilia remains to be determined. It has been shown that the motion of sensory hairs from the vestibular system could be controlled by exposing them to media that would induce contraction in muscle (Orman and Flock, 1983). These results imply that contractile mechanisms can influence the mechanics of sensory hairs, and that this contractile machinery may be susceptible to noise trauma. Cuticular plate changes have been observed after noise exposure showing 'softening' and bulging (Lindemann and Bredberg, 1972; Lim and Dunn, 1979). The stereocilia, being stiff rod-like structures pivot around the rootlet region (Flock, 1977) which is a region most likely to absorb stress during overstimulation. A denaturing of the molecular structure in the rootlet region after stimulation has been reported (Tilney et al., 1982). Furthermore, fractured rootlets as viewed with transmission electron microscopy are not uncommon findings after noise exposure (Engström et al., 1983, 1986).

In the present investigation it was found that the IHC stereocilia threshold change did not show further shifts with increasing exposure duration. This is in contrast to the ABR threshold shifts which showed a dependent change in sensitivity with increased duration of exposure. The specific cause for this discrepancy remains unknown. It is obvious that at number of other factors besides the threshold of the IHC stereocilia can be affecting the $\mathrm{ABR}$ threshold response. Another discrepancy between the electrophysiological response and the stereocilia mechanics was seen when the location of damage was compared. Five weeks after the exposure, the IHC stereocilia in the apical regions of the cochlea were normal whereas the electrical response continued to show a $25 \mathrm{~dB}$ shift in threshold sensitivity. Preliminary light microscopic findings show that at this time there is extensive swelling of the afferent dendrites beneath the inner hair cells. The location of the swollen synapses extend throughout the second, third and fourth turns of the cochlea. In addition, scattered OHC loss is found in the upper cochlear turns. These morphological changes would certainly contribute to the persisting ABR threshold shifts that are seen after the 5 -week recovery period. A correlation between dendritic swelling and compound action potential threshold shifts have been reported (Goulios and Robertson, 1983). However, additional electrophysiological studies are needed such as single unit recordings, $N_{1}$ latency, and $\mathrm{N}_{1}$ masked tuning curves in order to gain a better understanding of the physiological response and how it is correlated to the stereocilia changes.

One important question concerning stereocilia pathology induced by noise trauma has been whether or not the effect is reversible. Tilney et al. (1982) showed a reversible decrease in the number of cross-bridges between adjacent actin filaments after inducing a TTS in the alligator lizard. Mulroy and Whaley (1984) have shown a reversible clumping of the stereocilia during a noise-induced TTS. The findings in this study indicate that the IHC stereocilia mechanical properties can also recover.

In conclusion, the relationship of the stereocilia changes to the partial recovery of the ABR threshold sensitivity leads us to suggest that the temporary component of the threshold shift may be partly due to the observed increase in IHC stereocilia threshold, while the permanent $A B R$ sensitivity loss is in part a consequence of the $\mathrm{OHC}$ loss and in part due to the swollen afferent dendrites seen beneath the IHCs.

\section{Acknowledgements}

The authors are grateful for the comments offered by Günter Rösler and the secretarial help of Katarina Florin. Supported by grants from the Swedish Medical Research Council 04x-0246: The Foundation Tysta Skolan; The Torsten and Ragnar Söderberg Foundation; and the Swedish Work Environmental Fund.

\section{References}

Borg. E. and Engström, B. (1983) Hearing thresholds in the rabbit: A behavioral and electrophysiological study. Acta Oto-Laryngol. 95, 19-26. 
Davis, H., Morgan, C.T., Hawkins, J., Galambas, R. and Smith, F.W. (1946) Temporary deafness following exposure to loud tones and noise. Laryngoscope 56, 19-21.

Engström, B. and Borg, E. (1981) Lesions to cochlear inner hair cells induced by noise. Arch. Oto-Rhino-Laryngol. 230, 279-284.

Engström, B., Flock, Å. and Borg, E. (1983) Ultrastructural studies of stereocilia in noise-exposed rabbits. Hear. Res. $12,251-264$.

Engström, B., Borg, E. and Canlon, B. (1986) Morphology of stereocilia on cochlear hair cells after noise exposure. In: Applied and Basic Aspects of Noise-Induced Hearing Loss, pp. 30-41. Editors: D. Henderson, R. Salvi and D. Hamernik, Plenum Press Inc., New York.

Flock, $\AA$. (1977) Sensory transduction in hair cells. In: Handbook of Sensory Physiology, Vol. 1. Principle of Sensory Physiology, pp. 396-441. Editor: W.R. Lowenstein. Springer-Verlag, New York.

Flock, A. and Strelioff, D. (1984) Studies on hair cells in isolated coils from the guinea pig cochlea. Hear. Res. 15 , $11-18$.

Goulios, H. and Robertson, D. (1983) Noise-induced cochlear damage assessed using electrophysiological and morphological criteria: An examination of the equal energy principle. Hear. Res. 11, 327-341.

Hirokawa, N. and Tilney, L.G. (1982) Interactions between actin filaments and between actin filaments and membranes in quick-frozen and deeply etched hair cells of the chick ear. J. Cell Biol. 95, 249-261.

Leibovitz, A. (1963) The growth and maintenance of tissue cell cultures in free gas exchange with the atmosphere. Am. J. Hyg. 78, 173.

Liberman, M.C. and Dodds, L.W. (1984) Single-neuron labeling and chronic cochlea pathology. III. Stereocilia damage and alterations of threshold tuning curves. Hear. Res. 16, $55-74$.

Liberman, M.C. and Kiang, N.Y.S. (1978) Acoustic trauma in cats: Cochlear pathology and auditory nerve activity. Acta Oto-Laryngol. Suppl. 358, 1-63.

Lim. D.J, and Dunn, D.E. (1979) Anatomic correlates of noise induced hearing loss. Otolaryngol. Clin. N. Am. 12, $493-513$.

Lindemann, H.H. and Bredberg, G. (1972) Scanning electron microscopy of the organ of Corti after intense auditory stimulation: Effects on stereocilia and cuticular surface of hair cells. Arch. Klin. Exp. Ohren, Nasen, Kehlkopfheilkd. 203, 1-15.

Pickles, J.O., Osborne, M.P. and Comis, S.D. (1987) Vulnerability of tip links between stereocilia to acoustic trauma in the guinea pig. Hear. Res. 25, 173-184.

Mulroy, M.J. and Whaley, E. (1984) Structural changes in auditory hairs during temporary deafness. Scan. Electron Microsc. 1I, 831-840.

Orman, S. and Flock, А. (1983) Active control of sensory hair mechanics implied by susceptibility to media that induce contraction in muscle. Hear. Res. 11, 261-266.

Robertson, D. (1982) Effects of acoustic trauma on stereocilia structure and spiral ganglion cell tuning properties in the guinea pig cochlea. Hear. Res. 7, 55 74 .

Saunders, J.C. and Flock, A. (1986) Recovery of threshold shift in hair-cell stereocilia following exposure to intense stimulation. Hear. Res. 23, 233-244.

Saunders, J.C., Canlon, B. and Flock, A. (1986a) Growth of threshold shift in hair-cell stereocilia following overstimulation. Hear. Res. 23, 245-256.

Saunders, J.C.. Canlon, B. and Flock, A. (1986b) Mechanical changes in stereocilia following overstimulation: Observations and possible mechanisms. In: Applied and Basic Aspects of Noise-Induced Hcaring Loss, pp. 11-29. Editors: D. Henderson, R. Salvi and D. Hamernik, Plenum Press Inc., New York.

Slepecky, N., Hamernik, R., Henderson, D. and Coling, D. (1982) Correlation of andrometric data with changes in cochlear hair cell stereocilia resulting from impulse noise trauma. Acta Oto Laryngol. 93, 329-340.

Slepecky, N. and Chamberlain, S.C. (1982) Actin in cochlear hair cells: Implications for stereocilia movement. Arch. Oto-Rhino-Laryngol. 234, 131-134.

Strelioff, D. and Flock, Å. (1984) Stiffness of sensory-cell hair bundles in the isolated guinea pig cochlea. Hear. Res. 15. 19-25.

Thorne, P.R., Gavin, J.B. and Herdson, P.B. (1984) A quantitative study of the sequence of topographical changes in the organ of Corti following acoustic trauma. Acta OtoLaryngol. 97, 69-81.

Tilney, L.G., Saunders, J.C., Engelman, E. and DeRosier, D.J. (1982) Changes in the organization of actin filaments in the stereocilia of noise-damaged lizard cochlea. Hear. Res. 7 , 181-197. 